\title{
Analysing Discursive Communities and Semantic Networks on Twitter: An Entropy-Based Approach †
}

\author{
Tommaso Radicioni ${ }^{1,2}$, Tiziano Squartini ${ }^{2}$ and Fabio Saracco ${ }^{2}$ \\ 1 Scuola Normale Superiore, Pisa, Italy \\ 2 IMT-School For Advanced Studies, Lucca, Italy \\ + Presented at the Entropy 2021: The Scientific Tool of the 21st Century, 5-7 May 2021; Available online: \\ https://sciforum.net/conference/Entropy2021/.
}

Published: 5 May 2021

At the intersection between social sciences and network theory, the aim of this presentation is that of illustrating an entropy-based, data-driven approach to infer communities of Twitter users and looking at their interactions within a specific discussion. Amongst the various kind of interactions, the retweets have been chosen as a particularly insightful relational mechanism featured by Twitter. Our approach is based on a Shannon entropy maximization under certain constraints which guarantees that the procedure is unbiased and suitable for being applied to any properly-defined Twitter discussions.

One of the main results of our analysis is the operational definition of "discursive communities" as groups of users who share significantly-similar retweeting patterns. Comparing the observed bipartite network of users retweeting activities with the outcome of a properly-defined benchmark model, i.e., the Bipartite Configuration Model (BiCM), our inference method validate the similarity in the online activity of Twitter users who share the same contents, published by the accounts of public figures, to infer the presence of users communities with supposedly common features. The discursive communities recovered by our method display a coherent picture of its users behavior, in terms of retweeting and mentioning activities; besides, these communities are consistent with the political coalitions and sensitive to the dynamics characterizing the relationship within and between these coalitions as well.

A second core result of our analysis concerns the study of the mechanisms that shape the Twitter discussions characterizing the aforementioned discursive communities. By monitoring, on a daily and a monthly basis, the structural evolution of the community-generated semantic networks, the users in each community are observed to be characterized by a significantly different online behavior, thus inducing semantic networks with diverse topological structures.

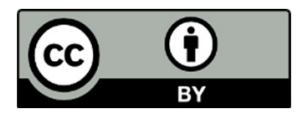

(C) 2021 by the authors. Licensee MDPI, Basel, Switzerland. This article is an open access article distributed under the terms and conditions of the Creative Commons Attribution (CC BY) license (http://creativecommons.org/licenses/by/4.0/). 